\title{
Cold atom-ion experiments in hybrid traps
}

\author{
Arne Härter ${ }^{1}$ and Johannes Hecker Denschlag ${ }^{1}$ \\ ${ }^{1}$ Institut für Quantenmaterie and Center for Integrated Quantum Science and Technology IQ ${ }^{S T}$, Universität Ulm, 89069 Ulm, Germany
}

\begin{abstract}
In the last 5 years, a novel field of physics and chemistry has developed in which cold trapped ions and ultracold atomic gases are brought into contact with each other. Combining ion traps with traps for neutral atoms yields a variety of new possibilities for research and experiments. These range from studies of cold atom-ion collisions and atom-ion chemistry to applications in quantum information science and condensed matter related research. In this article we give a brief introduction into this new field and describe some of the perspectives for its future development.
\end{abstract}

\section{INTRODUCTION}

The two research fields of trapped ultracold atoms and trapped laser-cooled ions have undergone a spectacular development during the last decades. They can be viewed as two cornerstones of today's research on quantum physics and they are continuing to push the limits of this field [1, 2, 3, 4, 5]. Only quite recently, however, have trapping technologies for ions and neutral atoms been combined in hybrid setups to allow for the controlled immersion of ions into clouds of ultracold atoms [6, 7, 8].

Traps for ions and traps for neutral atoms have complementary properties that turn hybrid atom-ion setups into versatile and powerful experimental tools. On the one hand, Paul traps for ions can be tight and several $\mathrm{eV}$ deep, allowing for a strong localization of the ions and for almost arbitrarily long storage times. On the other hand, even in such tight traps, laser-cooled ions are typically separated spatially by several micrometers due to their electrostatic repulsion. Thus, they can be individually optically detected and addressed. A single ion can then be employed as a well localized probe, e.g., to investigate local properties of an atomic cloud. In contrast, neutral atoms do not interact nearly as strongly as their charged counterparts. They can thus be prepared in large, dense ensembles consisting of millions of particles. Evaporative cooling methods provide access to temperatures on the nano-kelvin scale and to Bose-Einstein condensation. Ultracold clouds of atoms therefore lend themselves to sympathetic cooling of ions. Furthermore, they can also be used as sensitive bolometers to measure energy deposited by single ions, as even small atomic temperature changes can be detected. These first examples already give an impression of how the atomic and ionic systems complement each other and how they combine to form a fascinating experimental playground.

In this article we provide an introduction into the field of cold atom-ion experiments. We intend to give the reader a feeling for the characteristics of atom-ion interactions, highlight the most important experimental factors and give a rough overview of the current status of research activities. Finally, we also briefly discuss some possible future directions of the field. The discussions within this review should not be considered a complete summary of the field and the reader is encouraged to further reading starting with the references given here. This article is organized as follows: In section 2 we study the $1 / r^{4}$ interaction potential between an ion and an atom. It is much more long-range than the $1 / r^{6}$ van-der-Waals potential between neutral ground state atoms, but of shorter range than the $1 / r^{3}$ interaction between dipoles. In section 3 we give an overview of typical experimental realizations of atom-ion hybrid traps. While atom and ion traps are in principle wellcompatible, ion micromotion in the Paul trap represents an experimental obstacle for atom-ion experiments in the lowest energy regime. As discussed in section 4 in the context of elastic atom-ion collisions, this trap-driven micromotion leads to heating effects and sets a lower limit to the attainable collision energies. In section 5 we then turn to cold chemical reactions between atoms and ions, which is a vast field of research. Here, we constrain ourselves to the recent developments with respect to cold reactions in hybrid traps. One important reaction is charge transfer where the positive charge of the ion is passed onto an atom of the neutral atom gas. Furthermore, we also touch upon quenching collisions and the formation of charged molecules. Finally, in section 6 we review some future prospects for atom-ion experiments. On the one hand, we cover ideas and first experiments in the direction of quantum information science. On the other hand, prospects in the context of condensed matter physics are discussed. In particular, we consider charge transport in a quantum gas, impurity physics, and possibilities of emulating solid state physics.

\section{THE ATOM-ION INTERACTION}

The atom-ion interaction potential is dominated by the effect of the electric field $\varepsilon(r)=e /\left(4 \pi \varepsilon_{0} r^{2}\right)$ of the ion on the polarizable atom. Here, $e$ is the elementary charge, $\varepsilon_{0}$ the vacuum permittivity and $r$ the separation between atom and ion. The electric field induces a dipole moment $p(r)=4 \pi \varepsilon_{0} \alpha \varepsilon(r)$ in the atom which has a polarizability $\alpha$. Due to the inhomogeneity of the field, the induced dipole feels an attractive force towards the ion. As a result, the atom-ion interaction is described by the polarization potential

$$
V(r)=-\frac{1}{2} p(r) \varepsilon(r)=-\frac{C_{4}}{r^{4}}
$$

with $C_{4}=\alpha e^{2} /\left(8 \pi \varepsilon_{0}\right)$. In addition to this scattering potential, the angular momentum potential $l(l+1) \hbar^{2} / 2 \mu r^{2}$ has to be considered, where $l$ is the angular momentum quantum number and $\mu$ is the reduced mass of the atom-ion system. As a consequence, for $\mathrm{p}$-wave scattering $(l=1)$ the resulting effective potential exhibits a barrier (see Fig.11). It defines two useful scales, namely a length scale $R^{*}=\sqrt{2 \mu C_{4} / \hbar^{2}}$ and an en- 


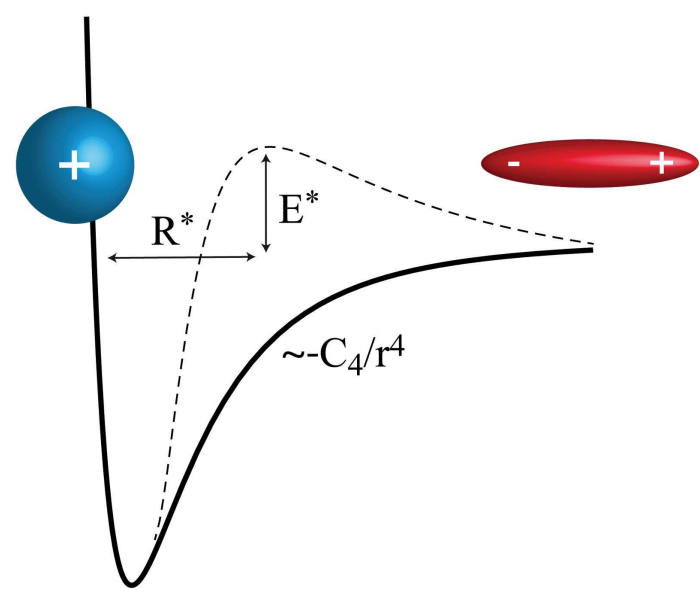

FIG. 1. The atom-ion polarization potential. The centrifugal barrier of the p-wave scattering channel (dashed line) leads to an energy scale $E^{*}$ and a length scale $R^{*}$.

ergy scale $E^{*}=\hbar^{2} /\left(2 \mu R^{* 2}\right)$. The length scale $R^{*}$ determines an interaction range and sets a typical value for the scattering length $a$. For the example of a ${ }^{138} \mathrm{Ba}^{+}$ion colliding with a ${ }^{87} \mathrm{Rb}$ atom, $R^{*}$ amounts to $R_{\mathrm{BaRb}}^{*}=295 \mathrm{~nm}$. This is about two orders of magnitude larger than the typical range (or scattering length) for van der Waals potentials between neutral atoms. The characteristic energy $E^{*}$ indicates the onset of the s-wave regime. For $\mathrm{Ba}^{+}$and $\mathrm{Rb}$ we get $E_{\mathrm{BaRb}}^{*}=50 k_{\mathrm{B}} \times \mathrm{nK}$, which is about three orders of magnitude lower as compared to the neutral case. As we will explain in more detail later in the text, typical atom-ion collision energies are currently technically limited to values on the order of $\mathrm{mK} \times k_{\mathrm{B}}$ or higher. Thus, reaching the s-wave scattering regime which would allow for control of atom-ion interactions remains one of the challenges of the field.

To describe elastic atom-ion collisions in the currently accessible higher energy regime one needs to account for contributions of many partial waves. A corresponding semiclassical expression for the elastic scattering cross section $\sigma_{\mathrm{e}}$ is given by [9]

$$
\sigma_{\mathrm{e}} \approx 5.08\left(\frac{\mu C_{4}^{2}}{\hbar^{2}}\right)^{1 / 3} E_{\mathrm{col}}^{-1 / 3}
$$

where $E_{\text {col }}$ denotes the collision energy. Thus, the semiclassical atom-ion cross section is energy-dependent and increases with decreasing energy. Elastic atom-ion collisions can be roughly grouped into two categories. 1) The glancing collisions in which particles are only slightly deflected and are preferably scattered in forward directions (see Fig.22). These collisions are prevalent [10, 11] and they are observable e.g. in ion-induced heating effects in ultracold atomic clouds. 2) The second category are the Langevin collisions in which two particles undergo a "hard" collision where large momentum/ energy transfer is possible. The Langevin collisions can be viewed in a simple classical picture (see Fig.22). We consider an atom-ion collision with an impact parameter $b$. If the impact parameter is smaller than a critical value

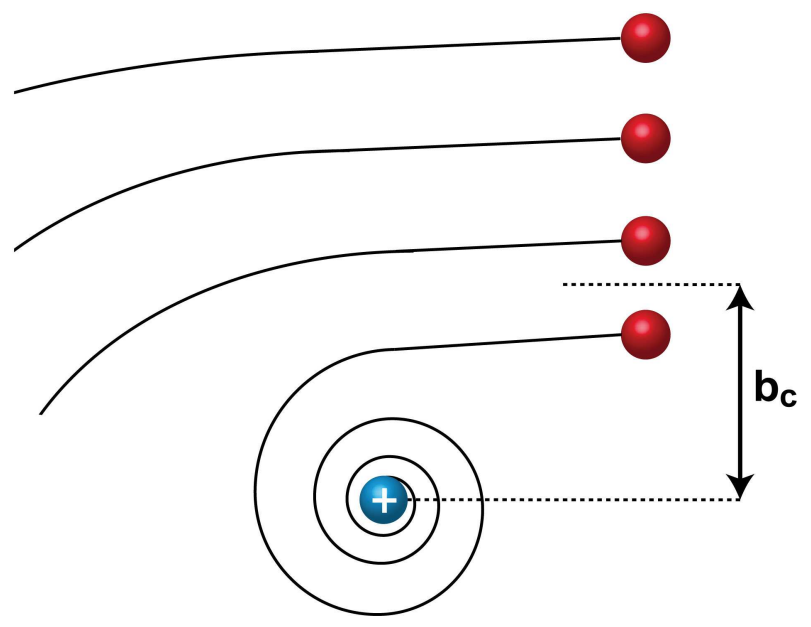

FIG. 2. Illustration of atom-ion collisions. Most collisions only lead to weak deflection of the collision partners in so-called glancing collisions. However, at impact parameters below the critical value $b_{c}$, the attraction between atom and ion becomes so strong, that the collision partners enter into an inward-spiraling trajectory. This results in a hard collision with a close encounter of the two nuclei.

$b_{c}=\left(2 C_{4} / E_{\mathrm{col}}\right)^{1 / 4}$, the two collision partners fall onto each other in a spiraling motion. As already stated, significant amounts of energy and momentum can be transferred between the collision partners and the direction into which the particles are scattered is isotropic. In such a Langevin collision a reaction can also occur, e.g. in which the charge is transferred from the ion to the atom or a molecule is formed. In fact, as reactions of this sort usually do require a close encounter of the two nuclei they will not occur in a glancing collision. The Langevin cross section is given by [9, 12]

$$
\sigma_{\mathrm{Lgvn}}=\pi \sqrt{2 C_{4}} E_{\mathrm{col}}^{-1 / 2} .
$$

Considering a collision between $\mathrm{Rb}$ and $\mathrm{Ba}^{+}$at an energy of about $1 \mathrm{mK} \times k_{B}$, the Langevin cross section $\sigma_{\mathrm{Lgvn}}$ is about 10 times smaller than $\sigma_{\mathrm{e}}$. A detailed introduction into the theory of atom-ion collisions, extending into the ultracold domain of s-wave scattering, can be found in refs. [13] and [14].

\section{EXPERIMENTAL REALIZATIONS AND THEIR IMPLICATIONS}

A challenge of hybrid atom-ion setups is to integrate overlapping traps for ions and atoms into a single apparatus. Depending on what kind of experiments are planned, the setups will look somewhat different. Currently, all groups in the field use Paul traps to confine the ions. However, the specifics of the design and in particular the dimensions of the Paul traps depend on the type of atom trap that is employed, in particular the magneto-optical trap (MOTs) [15, 16, 17], the faroff-resonant optical trap or the magnetic trap [18, 19, 20] (see Fig.3). 

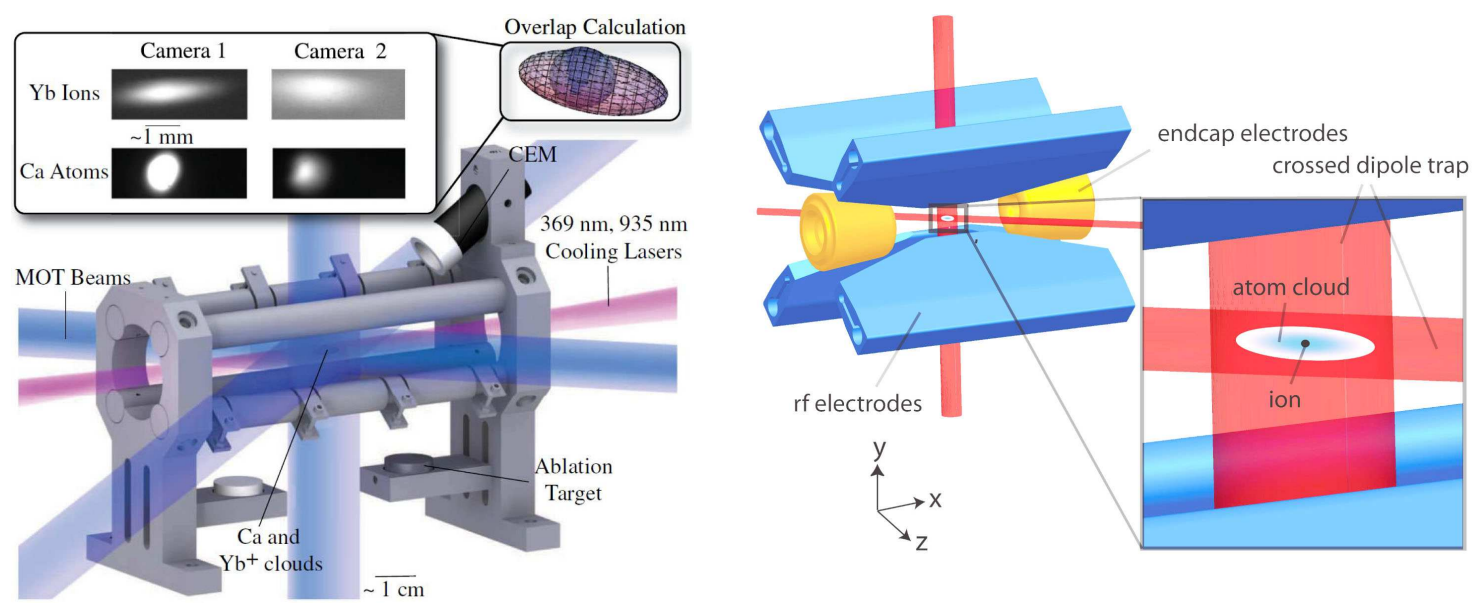

FIG. 3. Examples of hybrid atom-ion traps. Left: A combined MOT and ion trap featuring large separations of the Paul trap rf electrodes to allow for sizable MOT cooling beams inside the trapping volume of the Paul trap. Right: Alternative approach employing a crossed dipole trap for atomic confinement and a significantly smaller spacing of the rf electrodes. Images taken from [21] and [7].

When working with a MOT, relatively large laser beams with diameters of several $\mathrm{mm}$ are required. Thus, the Paul traps in such setups feature large spacings between their radiofrequency (rf) electrodes on the order of $1 \mathrm{~cm}$. Large Paul traps have the advantage that they allow for loading large ion crystals with hundreds or thousands of ions. These ion crystals can then be overlapped with the MOT atom clouds consisting of roughly $10^{6}$ atoms at temperatures around $100 \mu \mathrm{K}$ and densities of about $10^{9} \mathrm{~cm}^{-3}$. Such a setup is well suited for investigations of chemical reactions such as charge transfer or molecule formation in the cold regime. Measurements can be carried out in a continuous way, monitoring the fluorescence signals of the MOT and the trapped ions. However, in a MOT a good fraction of the atoms are in an excited electronic state which complicates the analysis of experimental signals.

For experiments where the atoms need to be in their electronic ground state, at low atomic temperatures or at high atomic densities, a MOT is not sufficient anymore. Instead, the atoms need to be trapped either in a magnetic trap or a far-off-resonance optical dipole trap. These atom traps allow for smaller ion traps (e.g. on the order of $1 \mathrm{~mm}$ ) which yield tighter ionic confinement. This comes at the price that the atomic cloud has to be prepared elsewhere and subsequently transported to the location of the ion trap. For example, in our atom-ion apparatus in Ulm we first prepare an ultracold atomic cloud at a temperature of $1 \mu \mathrm{K}$ in a magnetic trap using evaporative cooling. Afterwards, we transport the atoms over a distance of $30 \mathrm{~cm}$ to the ion trap, using a moving optical trap [18].

Working with a single ion (as compared to Coulomb crystals consisting of many ions) generally improves the control over the ion, e.g. in terms of its position, kinetic energy and internal state. On the downside, using single ions can be timeconsuming when rare events need to be investigated with significant statistics.
Ion traps and optical dipole traps for neutral atoms are in principle quite compatible with each other. The inhomogeneous electric fields $\varepsilon_{\text {trap }}$ of the ion trap only lead to a relatively weak deconfining anti-trapping potential $\propto 0.5 \alpha \varepsilon_{\text {trap }}^{2}$ for neutral atoms with a static polarizability $\alpha$. For Rb in our setup the corresponding "anti-trap" frequency is on the order of a few Hz. This is much smaller than the trap frequency of a typical optical dipole trap which ranges around one hundred Hz. Similarly, the influence of the optical trap on the ions is small compared to the deep Paul trap with its typical trapping frequencies on the order of $100 \mathrm{kHz}$.

An important experimental issue in a hybrid atom-ion setup is the micromotion in the Paul trap [22]. This is linked to the fact that, strictly speaking, the confining potential of a Paul trap is not conservative as it is based on oscillating electric fields. On top of the ion's so-called secular motion in the effective harmonic trapping potential, the ion also carries out a fast oscillatory motion (typically in the $\mathrm{MHz}$ range), the micromotion. The micromotion oscillations are a direct consequence of the oscillating electrical forces of the rf field on the ion. Figure 4 shows typical ion trajectories in one dimension in a Paul trap. The upper blue trace shows the motion of an ion oscillating symmetrically about the trap center. As the rf fields vanish at the center and increase when moving outwards, micromotion oscillations are strongest at the turning points of the harmonic secular motion. Technical imperfections of the ion trap can further enhance micromotion, leading to the so-called excess micromotion. For example, a stray electric offset field can shift the equilibrium position of the trapped ion, as illustrated by the lower red trace of the ionic trajectory. Furthermore, a phase delay of the rf voltage on opposite Paul trap electrodes also leads to excess micromotion. Here, the rf node, where the rf fields vanish, oscillates in space. Excess micromotion can be minimized by compensating the electrical stray fields and phase delays.

In current atom-ion experiments the typical energy scale for excess micromotion is on the order of $1 \mathrm{mK} \times k_{B}$ or above so 


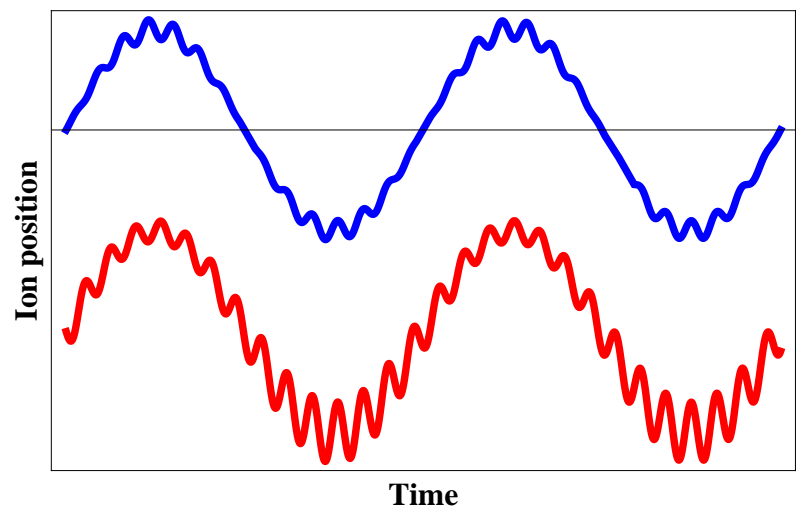

FIG. 4. Illustration of ion trajectories in a Paul trap (in the absence of a neutral atomic cloud). Upper trace: In an ideal configuration, the ion oscillates symmetrically about the nodal line of the rf field of the trap. Micromotion occurs predominantly at the turning points of the secular oscillations and vanishes when the ion passes through the rf node. Lower trace: An external electric field shifts the ion from the center of the trap. The ion experiences larger rf fields and thus undergoes excess micromotion oscillations. The energy contained in the excess micromotion can be much larger than the energy of the secular motion.

that it sets the dominant energy scale. In an atom-ion collision, the ionic micromotion energy can be transferred to the atom. The energy that the ion loses in such a collision will then quickly be replenished by the rf drive of the Paul trap. Thus, one effect of micromotion is that it leads to ion-induced heating of the neutral atomic cloud. It can also lead to atom loss if the atom-ion collision is strong enough that the atom leaves its trap.

\section{ELASTIC ATOM-ION COLLISIONS}

Collisions are elastic when the internal state of the colliding particles is not changed. As discussed in section 2, the elastic cross sections for atom-ion collisions are typically orders of magnitude larger than for cold neutral atoms due to the longer range of the $1 / \mathrm{r}^{4}$ potential. Such large cross sections are useful to reduce time constants for particle interactions. For example, possible applications are sympathetic cooling of ions with a cold atomic gas or fast atom-ion quantum gates. Furthermore, as we will discuss in section 6, strong atom-ion interactions are of interest in experiments with polaronic impurities aiming at the regime of strong coupling.

So far, experiments on elastic collisions between ions and cold atoms have been carried out with combinations of $\mathrm{Yb}^{+}$, $\mathrm{Ba}^{+}, \mathrm{Rb}^{+}, \mathrm{Ca}^{+}$, and $\mathrm{Rb}, \mathrm{Li}$ (see e.g. [7, 8, 20, 23, 24]). Typically, in these measurements one or more trapped ions are immersed into a trapped gas of about $10^{5}$ cold atoms. Elastic collisions between an ion and atoms lead to: 1) heating of the atomic cloud, 2) atom losses of the cloud, and 3) cooling or even heating of the ion. The collision rate $\Gamma_{e}$ is given by

$$
\Gamma_{e}=n_{\mathrm{at}} \sigma_{e} v_{\mathrm{ion}}
$$

where $v_{\text {ion }}$ is the velocity at which the ion moves through the atom cloud and $n_{\text {at }}$ is the local atomic density. From measurements of the decay of the atom number and the evolution of the atomic temperature one can obtain information on the cross sections and the kinetics of the ions.

A large ensemble of ultracold atoms at $\mathrm{nK}$ temperatures constitutes a buffer gas that can be employed as a coolant for ionic collision partners. Indeed, various experiments have demonstrated this atom-ion sympathetic cooling. In these experiments the kinetic energy of the single ion needs to be measured. Currently, the most precise methods for doing this are based on resolved-sideband cooling techniques [25]. However, as these techniques are quite involved, they have not yet been applied to measure ion energies following atom-ion collisions. Rather, a simpler but less precise temperature measurement, termed ion recooling, was used to show cooling of ions in an ultracold gas from much hotter temperatures to below $1 \mathrm{~K}$ [8](see also [23]). Ion recooling measures the time it takes to laser cool an ion to Doppler temperatures while observing its fluorescence. Yet another (but more indirect) method to measure ion temperatures involves atom-ion collisions. Given the cross section and the atomic density one can infer the ion velocity from observed collision rates. In an experiment with a single ${ }^{87} \mathrm{Rb}^{+}$ion and cold ${ }^{87} \mathrm{Rb}$ atoms, sympathetic cooling from several Kelvin down into the sub-mK regime was demonstrated [26, 27]. This result seems promising for a wide applicability of ultracold buffer gas cooling, as ${ }^{87} \mathrm{Rb}^{+}$is not amenable to laser cooling.

The question is how far down (beyond the mK-regime) one can sympathetically cool ions using ultracold atoms in a hybrid trap. Let us consider a single trapped ion which initially has been laser cooled to Doppler temperatures of about $1 \mathrm{mK}$. This ion is immersed into a cold atomic cloud with a temperature below $1 \mu \mathrm{K}$. Ignoring micromotion for a moment, one would expect the ion to be sympathetically cooled down to the temperatures of the atoms. Micromotion, however, prevents the ion from coming to rest but keeps it at kinetic energies that are set by the excess micromotion. One key to low ion energies is thus to minimize excess micromotion. This can be done with the help of atoms as we will discuss next.

On average, in a collision with an atom, the ion pumps energy into the atomic cloud, heating it up and leading to atom loss. This has been observed, e.g. in [24] (see Fig.5]. Here, a single $\mathrm{Rb}^{+}$ion is placed at the center of a $\mathrm{Rb}$ atom cloud. At a density of about $n_{\text {at }}=10^{11} \mathrm{~cm}^{-3}$ a continuous atom loss and heating effect on timescales of several seconds are observed. When excess micromotion is reduced (by compensating the stray electric fields $\varepsilon$ ), the mean kinetic energy of the ion decreases. This lowers heating and losses of the atomic cloud. It is instructive to consider the micromotion energies $E_{\text {eMM }}$ involved in this measurement. If we ignore the contribution to micromotion due to phase differences we can calculate the ion excess micromotion energy due to stray electric fields [22]. For the well-compensated case $(\varepsilon<0.1 \mathrm{~V} / \mathrm{m})$ we get $E_{\mathrm{eMM}}=10 \mu \mathrm{K} \times k_{\mathrm{B}}$ while in the electric field of $4 \mathrm{~V} / \mathrm{m}$ we get $E_{\mathrm{eMM}}=20 \mathrm{mK} \times k_{\mathrm{B}}$, an increase by more than three orders of magnitude. The sensitivity of the atomic loss on the ion energy can be used to directly minimize micromotion. Figure6 


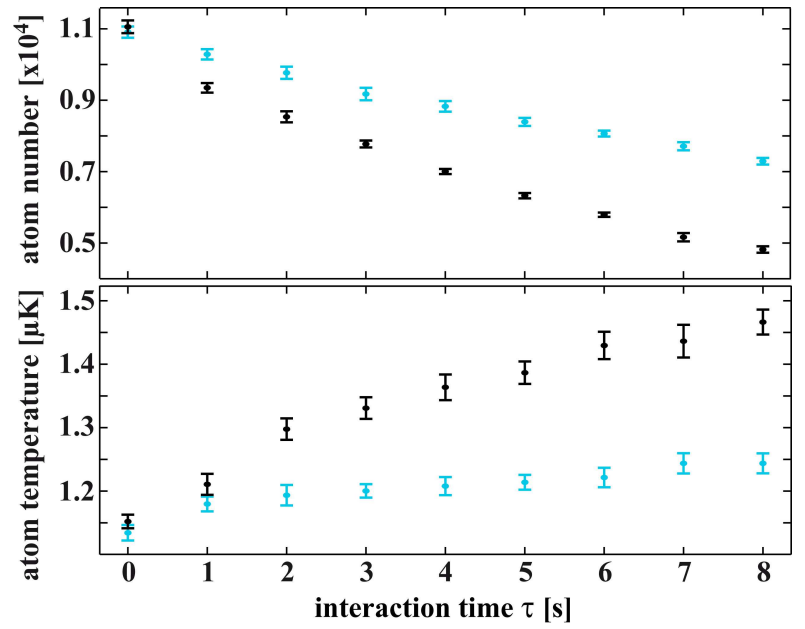

FIG. 5. As a consequence of the driven ion micromotion, a $\mathrm{Rb}$ ion induces continuous atom loss (upper panel) and heating (lower panel) in an ultracold $\mathrm{Rb}$ atom cloud. The measurement was performed for electric bias fields compensated to better than $\varepsilon<0.1 \mathrm{~V} / \mathrm{m}$ (blue data points) and a deliberately applied offset field of about $4 \mathrm{~V} / \mathrm{m}$ (black data points). Figure taken from [24].

shows the remaining atom number as a function of the electric offset field after an ion has been immersed for $\tau=8 \mathrm{~s}$. The peak in the atom number indicates the best compensation setting. By performing a simple fit to the data, the electric field can be minimized to better than $0.02 \mathrm{~V} / \mathrm{m}$, corresponding to excess micromotion energies (due to stray electric fields) below $1 \mu \mathrm{K} \times k_{\mathrm{B}}$. This result is comparable to the most precise micromotion compensation methods based on ion fluorescence signals [22, 24, 28, 29, 30, 31].

It needs to be pointed out that even when micromotion due to both stray electric fields and phase difference is compensated down to the $\mu \mathrm{K}$ level the mean kinetic energy of the ion can still be much larger. Besides anomalous heating effects [32, 33] where electrical field fluctuations from the trap electrodes heat up the ion, collisional effects between atoms and ions also lead to heating. This is discussed in the following two sections.

A more refined theoretical analysis [11, 34, 35] of the atomion dynamics in a trap shows that the energy distribution of the ion is non-thermal as it features a high energy tail. The prominence of the high energy tail depends critically on the atom-ion mass ratio. For a light ion interacting with much heavier atoms, the collision dynamics can even heat the ion to much higher "temperatures" than the one of the trapped atoms. This counterintuitive effect is due to a transfer of energy from micromotion into secular motion occurring in atomion collisions.

However, it turns out that as long as the ionic mass is larger than the mass of the neutral atom, the heating effect is moderate and the energy distribution is close to thermal. The average ionic energy can be then approximated by the plain excess micromotion energy (i.e. in the absence of the neutral atom gas) multiplied by a constant factor of order unity [11].

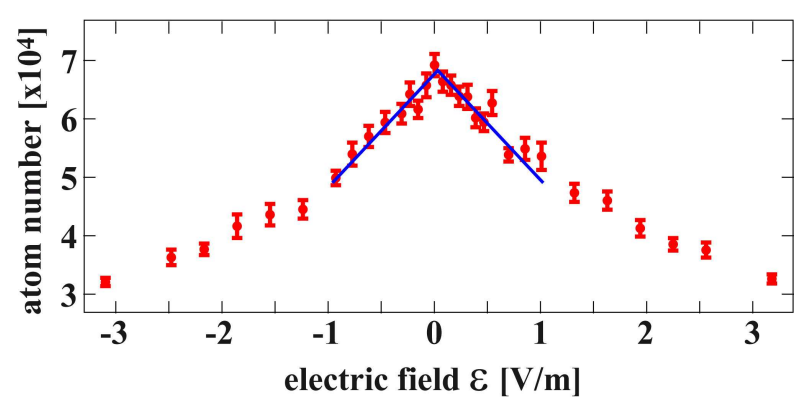

FIG. 6. Micromotion compensation. Shown is the remaining atom number of a cold atom cloud after an $8 \mathrm{~s}$ long interaction with a single ion for various electrical offset fields $\varepsilon$. A small $|\varepsilon|$ leads to small micromotion, minimizing atomic losses. Figure adapted from [24].

One further possible micromotion-induced heating mechanism is discussed in ref. [36]. In this case, ion excess micromotion is assumed to be perfectly compensated and the ion is initially at rest at the trap center. When this ion is approached by a neutral atom, the atom-ion interaction pulls the ion away from the trap center into regions of non-vanishing micromotion. Consequently, during the collision, the rf drive of the trap can pump energy into the system. The average amount of injected energy and thus the limit for the achievable collision energies depend on the trap parameters and on the atom-ion mass ratio. To minimize the heating effect a heavy ion (such as $\mathrm{Yb}^{+}$) in a gas of light atoms (such as Li) should be chosen.

Ultimately, micromotion-induced heating may set an effective limit on the collision energies that can be realistically achieved in a Paul trap environment. One viable path to circumvent such limitations may be the use of an optical dipole trap to trap ions. However, such trapping schemes are challenging as already small electric stray fields on the order of $0.1 \mathrm{~V} / \mathrm{m}$ exert comparatively strong forces on the ion which are hard to overcome with optical dipole traps. Despite these difficulties, a proof-of-principle experiment for optical trapping of an ion has been successfully carried out [37]. $\mathrm{A} \mathrm{Mg}^{+}$ ion, initially trapped in a linear Paul trap, was transferred into an optical dipole trap consisting of $190 \mathrm{~mW}$ of tightly focussed laser light (beam waist $\approx 7 \mu \mathrm{m}$ ) at a wavelength of $280 \mathrm{~nm}$. The achieved trap depth was about $40 \mathrm{mK} \times k_{\mathrm{B}}$ and the maximal confining force was on the order of $10^{-19} \mathrm{~N}$. Spontaneous scattering of light led to strong heating which limited the ion trap lifetime to about $2 \mathrm{~ms}$.

\section{INELASTIC COLLISIONS AND COLD CHEMICAL REACTIONS}

We now turn to inelastic collisions and reactions. We will first consider charge transfer where the charge of the ion "hops" onto the neutral collision partner. Next we discuss quenching collisions where internal states of atomic or molecular ions relax to energetically lower lying states via colli- 
sions with neutral atoms. Finally, we describe some first observations of cold atom-ion reactions in which molecules are formed.

\section{A. Charge exchange reactions}

In the charge exchange reactions described here, a trapped ion $A^{+}$collides with an ultracold atom $B$ to produce $A+B^{+}$. In this process, particle $A$ is lost and replaced by $B^{+}$which is now trapped in the deep ion trap. Such a process can be detected on a single particle level, e.g. via the loss of ion fluorescence signals [6, 7, 8] (see Fig.77). For example, in the experiments in Ulm, the fluorescence of a single laser-cooled $\mathrm{Ba}^{+}$ion is monitored. As soon as this ion undergoes a charge exchange with neutral $\mathrm{Rb}$, the ion is turned into a $\mathrm{Ba}$ atom and it stops fluorescing. The created $\mathrm{Rb}^{+}$ion does not scatter light in the visible and is "dark". Such a "dark" ion can be investigated via mass spectrometry in the Paul trap. This type of investigation can be done with a single ion or with many ions that form a crystal of "dark" and "bright" ions (see Fig.7). When the drive frequency of the trap is modulated at a secular frequency of a "dark" ion, this ion is strongly heated. Via Coulomb coupling between the "dark" and "bright" ions, the "bright" ions are sympathetically heated and their fluorescence signals become blurred or are even lost completely. This method is routinely used to analyze the contents of mixed ion crystals consisting of up to thousands of ions [17, 21] (see Fig.77).

Charge transfer can proceed in two different ways: (1) through emission of a photon $\gamma$ in a radiative charge transfer (RCT) $A^{+}+B \rightarrow A+B^{+}+\gamma$, or (2) in a non-radiative charge transfer (nRCT), $A^{+}+B \rightarrow A+B^{+}$. The probability for these charge exchange reactions to occur in a collision can vary by several orders of magnitude. It depends on the details of the colliding particles and their electronic states. For example, the probability for nRCT can be very large for the case of a crossing of energy levels of the two scattering channels $A^{+}+B$ and $A+B^{+}$(see Fig. 8 a).

Already a good number of charge exchange processes in cold atom-ion collisions have been studied, involving collisions of ions such as $\mathrm{Yb}^{+}, \mathrm{Ba}^{+}, \mathrm{Ca}^{+}, \mathrm{N}_{2}^{+}$with neutral atoms such as $\mathrm{Yb}, \mathrm{Rb}$ and $\mathrm{Ca}[6,7,17,21,38,39,40,41,42]$. In general, these experiments confirmed that the charge transfer rate coefficients are bounded from above by the classical Langevin collision cross section (see section $\amalg$ ). This seems reasonable when we assume that charge transfer requires a close encounter of ion and atom, i.e. a Langevin collision. Making use of its cross section we can calculate the corresponding collision rate

$$
\Gamma_{\mathrm{Lgvn}}=n_{\mathrm{at}} \sigma_{\mathrm{Lgvn}} v_{\mathrm{ion}},
$$

where the ionic velocity $v_{\text {ion }} \propto E_{\text {col }}^{1 / 2}$ is assumed to be much larger than the atomic velocity. With $\sigma_{\mathrm{Lgvn}} \propto E_{\mathrm{col}}^{-1 / 2}$ (see equation 3 we find that $\Gamma_{\text {Lgvn }}$ should be independent of the collision energy, a fact which was confirmed experimentally [6, 21, 38, 42, 43]. While bounded from above, charge exchange rates can be very much smaller than the Langevin collision rate. Suppression factors ranging from about 1 to $10^{-5}$ have been measured. The magnitude of the suppression factor is determined by the details and the mechanism of the collision, i.e. whether it involves emission of a photon and what the precise form of the relevant molecular potential energy curves is.

An exception to the applicability of Langevin theory was observed in investigations of reactions between $\mathrm{N}_{2}^{+}$molecular ions with $\mathrm{Rb}$ atoms in the excited ${ }^{2} P_{3 / 2}$ state [40]. In these measurements, the charge exchange rate was found to be 4 times larger than the maximal value allowed within Langevin theory. This large rate can be explained by taking into account the permanent electric quadrupole moment of the excited state of neutral $\mathrm{Rb}$ that interacts with the ion. This interaction adds to the polarization potential considered in Langevin theory and further increases the reaction cross section.

While charge exchange reactions provide an interesting field to explore, they are often undesired in atom-ion experiments. In order to avoid charge exchange completely, one can choose appropriate combinations of species (such as $\mathrm{Ba}^{+}$ and $\mathrm{Ca}[41,44]$ ) in which chemical reactions are prohibited energetically, at least in ground state collisions.

\section{B. Quenching reactions}

In addition to charge exchange, collisions of atomic (or molecular) ions with neutral atoms can lead to a relaxation of internal excitations in so-called quenching reactions. Quenching can be applied to sympathetically cool internal excitations in addition to the external motion (as previously discussed in section 4 ).

In ref. [39], $\mathrm{Yb}^{+}$ions were prepared in electronically excited metastable states and immersed into ultracold $\mathrm{Rb}$ atom clouds. Quenching in these experiments was detected by shining in resonant laser light, leading to ionic fluorescence signals as soon as the quench from the metastable states into the ground state ${ }^{2} S_{1 / 2}$ occurred. While the excitation into the ${ }^{2} F_{7 / 2}$ state of $\mathrm{Yb}^{+}$was apparently efficiently quenched, no such reaction was observed upon preparation of the ${ }^{2} D_{3 / 2}$ state. It will be interesting to understand the physical origin of these different quenching dynamics in future investigations. In experiments with molecular $\mathrm{BaCl}^{+}$ions immersed into a cloud of ultracold $\mathrm{Ca}$ atoms, quenching of internal vibrational excitations of the molecular ions was achieved [44]. This particular combination of atomic and ionic species was chosen because of the stability with respect to charge exchange reactions. Clouds of $\mathrm{BaCl}^{+}$ions were prepared in a Paul trap and their internal degrees of freedom equilibrated to the temperature of the surrounding vacuum chamber which was at room temperature. In this initial setting, the vibrational ground state of the molecules was populated with a probability of about $80 \%$. After immersion of these molecules into an ultracold cloud of $\mathrm{Ca}$ atoms produced in a MOT, the ground state population increased to $90 \%$, only limited by heating effects induced by black-body radiation from the vacuum chamber walls. The quenching rate constant $k_{q}=1 \times 10^{-9} \mathrm{~cm}^{3} \mathrm{~s}^{-1}$ was found to be large and it indicates that every fifth Langevin collision leads to a quench of the 

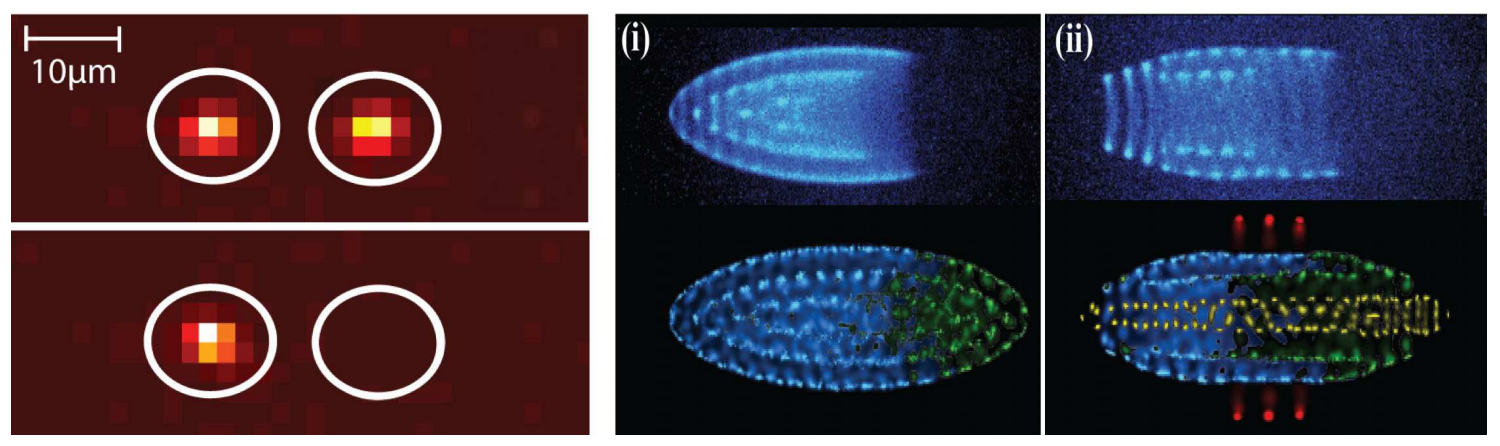

FIG. 7. Experimental signals of chemical reactions in hybrid traps. Left: Observing a charge exchange reaction in a string of two ions in a linear Paul trap. Initially, two ${ }^{138} \mathrm{Ba}^{+}$ions are laser cooled and then brought into contact with a ${ }^{87} \mathrm{Rb}$ atom cloud. When one ion turns dark, this is indication that a reaction has taken place. With subsequent measurements the mass of this dark ion can then be determined. Right: Charge exchange reactions using a large ion crystal with up to several hundred ions. (i) Experimental picture (upper image) and simulation (lower image) of a $\mathrm{Ba}^{+}$ion crystal before interaction. (ii) Images after interaction with ${ }^{87} \mathrm{Rb}$ atoms. In the simulation, ${ }^{138} \mathrm{Ba}$ ions are shown in blue, lighter $\mathrm{Ba}^{+}$isotopes in green, ${ }^{87} \mathrm{Rb}^{+}$ions in yellow and $\mathrm{BaRb}^{+}$molecular ions in red. By comparing the experimental pictures to the simulations, the product fractions after the reaction can be inferred. Figures taken from [7] and [17]
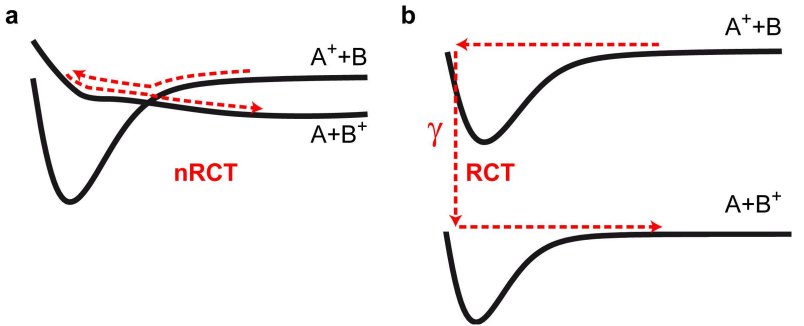

FIG. 8. Charge transfer. a) An avoided crossing of molecular potential curves can favor non-radiative charge transfer (nRCT) if the crossing is not fully adiabatic or non-adiabatic. b) Radiative charge transfer involves the emission of a photon $\gamma$.

vibrational excitation.

\section{Formation of charged molecules and molecular reactions}

The controlled formation of cold ionic molecules is an important goal in the field of atom-ion experiments. It is related to research of chemistry in interstellar clouds where molecules are formed under dilute (density $<10^{6} \mathrm{~cm}^{-3}$ ) and cold (temperature $\sim 10 \mathrm{~K}$ ) conditions [45, 46]. A large number of groups have contributed to this field with beautiful experiments with collision energies down to the low $\mathrm{K} \times k_{B}$ regime (see e.g. [47, 48, 49, 50]). Here, however, we will constrain ourselves to laboratory experiments in the $\mathrm{mK}$ regime. In this regime, to date only a few chemical reactions have been observed.

In reference [17, 42] the collision of $\mathrm{Ca}^{+}$and $\mathrm{Rb}$ was studied. It was found that fast reactions occur when the $\mathrm{Ca}^{+}$ions are excited to their $4 p$ state. As reaction products, both $\mathrm{Rb}^{+}$ due to charge transfer and $\mathrm{RbCa}^{+}$molecular ions were de- tected. These molecular ions were probably produced via radiative association, a process in which a photon is emitted upon the atom-ion collision and a molecular ion is formed: $A^{+}+B \rightarrow(A B)^{+}+\gamma$. Both the atomic and the molecular ions in these experiments were observed within a large ion crystal in the Paul trap. They were identified using the mass spectrometry methods discussed in section $\mathrm{VA}$

Another pathway to the formation of molecular ions is three-body recombination, where the ion and an atom combine to form a molecule and a second atom carries away part of the binding energy. In section $\Pi$ we discussed the large atom-ion interaction radius $R^{*}$ on the order of hundreds of nanometers. As a consequence, there is a significant probability for two atoms to enter the atom-ion interaction range simultaneously, even at moderate atomic densities of $10^{12} \mathrm{~cm}^{-3}$. Collisions involving a single ion and two neutral atoms thus become relevant at much lower densities than in the wellknown case of interatomic three-body collisions. In neutral atomic gases three-body recombination typically leads to the loss of all three atoms involved in the reaction [51, 52, 53] as the released binding energy kicks the particles out of trap. In the case of atom-ion three-body recombination, the ionic product will typically not be lost as it is retained in the very deep Paul trap. In an experiment with a $\mathrm{Rb}^{+}$ion in a cold $\mathrm{Rb}$ cloud [26] the energy released in a three-body recombination event was found to be on the order of a few $0.1 \mathrm{eV}$, much smaller than the ion trap depth. Although still trapped, the ion was ejected out of the $100 \mu \mathrm{m}$-sized atom cloud upon the recombination (see Fig.9). The ion's trajectory in the trap was so large that it barely interacted with the atomic sample at the trap center. Thus, for time periods of a few seconds, the atom-ion interaction effectively stopped, leading to a drastic reduction of atomic loss. Interestingly, the produced molecules could not be detected directly - at the end of each experiment exclusively an atomic $\mathrm{Rb}^{+}$ion was observed. As a consequence it could not be determined whether $\mathrm{Rb}_{2}$ or $\mathrm{Rb}_{2}^{+}$ 
a

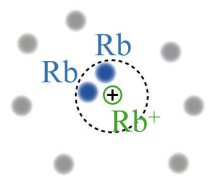

b

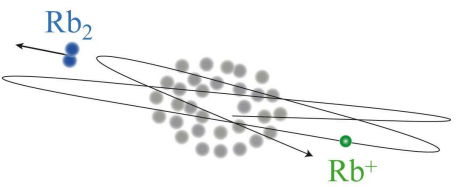

FIG. 9. Illustration of the effect of an atom-ion three-body collision. The large amount of released binding energy ejects the ion from the atom cloud and promotes it onto a large orbit within the ion trap. This effectively stops the atom-ion interaction for long time periods on the order of seconds.

molecules had been initially produced, as the $\mathrm{Rb}_{2}^{+}$molecules might have subsequently quickly disintegrated back to $\mathrm{Rb}^{+}$in another light assisted collision with $\mathrm{Rb}$ atoms.

The production of $\mathrm{Rb}_{2}^{+}$molecules was directly observed in another experiment [54]. The production path, however, starts with neutral atoms. A relatively dense atomic cloud of neutral $\mathrm{Rb}$ atoms $\left(10^{13} \mathrm{~cm}^{-3}\right)$ was placed inside a Paul trap. Via interatomic three-body recombination in this cloud, $\mathrm{Rb}_{2}$ molecules were formed. Some of these neutral molecules were then resonantly photoionized and subsequently trapped in the Paul trap where they were detected. Interestingly, when bringing these molecular $\mathrm{Rb}_{2}^{+}$ions back into contact with the optically trapped $\mathrm{Rb}$ gas they quickly dissociate (on the ms timescale) into atomic $\mathrm{Rb}^{+}$ions, as discussed in the previous paragraph. The dissociation mechanism is not yet fully understood and will be the subject of further investigations.

\section{FUTURE PROSPECTS FOR ATOM-ION EXPERIMENTS}

Hybrid systems of ions and atoms also lend themselves to experiments on topics of quantum information science and condensed matter physics. In the following we briefly discuss some theoretical schemes that have been proposed in these contexts.

\section{A. Atom-ion Feshbach resonances}

A key to the engineering of complex many-particle systems is the control over the interparticle interactions. Such control is most easily obtained in the regime of s-wave scattering where the interaction can be described by a single quantity, the scattering length. Similar to established techniques in neutral atomic gases, atom-ion Feshbach resonances could be used to tune the scattering length. The atom-ion interaction could thus be set strongly attractive to non-interacting to repulsive. Furthermore, Feshbach resonances would be convenient tools to efficiently form charged molecules in precisely defined quantum states. Indeed, such resonances have been predicted to exist in collisions of singly ionized alkaline earth atoms with alkali atoms, such as $\mathrm{Ca}^{+}+\mathrm{Na}$ [13] (see Fig.10). Interestingly, resonances should appear already at relatively low magnetic fields on the order of tens of Gauss $\left(1 \mathrm{G}=10^{-4} \mathrm{~T}\right)$. This can

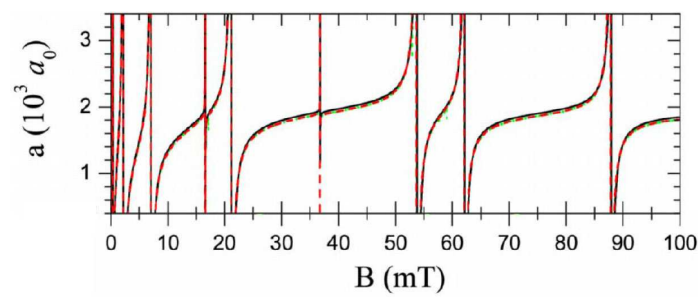

FIG. 10. Calculated Feshbach resonances for $\mathrm{Ca}^{+}$colliding with $\mathrm{Na}$. Shown is the scattering length $a$ versus the magnetic field $B$. Already at moderate magnetic fields below $10 \mathrm{mT}$ a number of resonances are predicted. Figure adapted from [13].

be explained as a consequence of the relatively long-range $1 / \mathrm{r}^{4}$ potential which leads to many weakly bound molecular states. In order to make use of Feshbach resonances, the swave regime needs to be reached, which typically corresponds to collision energies of $100 \mathrm{nK}-1 \mu \mathrm{K} \times k_{B}$. This is a challenge.

\section{B. Prospects for quantum information applications}

The great success of both ions and atoms in the context of quantum information science obviously raises hopes that a combined atom-ion system may provide additional opportunities for this field. Ions, on the one hand, are routinely controlled on the single particle level and their tight traps allow for fast and efficient transport schemes. Neutral atoms can exhibit long coherence times and large arrays of atoms can be prepared in an optical lattice with a single atom per lattice site, constituting a quantum register. An ion may then be used to transport quantum information between different sites of the optical lattice and to entangle atoms. Similarly, a single ion can also read out information so that it can act as a read and write head. The transfer of quantum information between the ion and the atoms could be achieved via controlled collisions where both the ion and the atom are held in their individual trap potentials [55]. A special situation arises here due to the long-range atom-ion interaction potential in combination with the tight trapping potentials. Depending on the distance between atom and ion, this can lead to trap-induced shape resonances or confinement-induced resonances. In both cases, vibrational trap states become equal in energy to molecular states giving rise to avoided crossings. It has been shown that this can be exploited to implement atomion quantum gates [56]. However, it must be said that ion micromotion severely complicates the atom-ion collisional processes and could make the experimental realization of the proposed schemes challenging [57].

In ref. [58] an ion is proposed to act as a quantum switch for the transport of neutral atoms between lattice sites. It is conjectured that the switch can be made state dependent which can be used to create controlled entanglement of atomic and ionic states.

Experimentally, the degree of control over atom-ion systems to realize such quantum information schemes has not been 

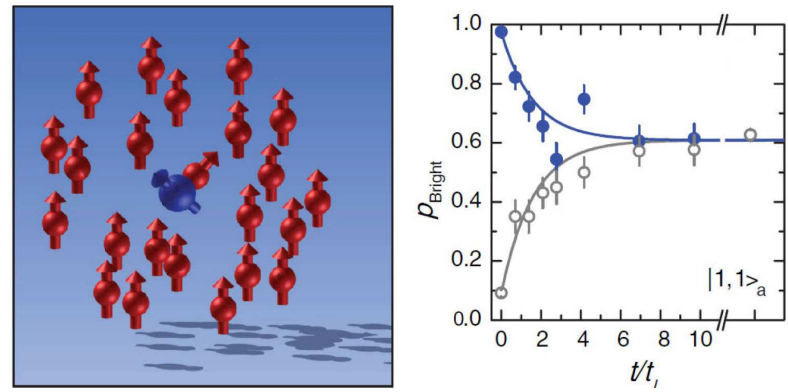

FIG. 11. A single $\mathrm{Yb}^{+}$ion as an impurity in a spin-polarized atomic gas. The ${ }^{174} \mathrm{Yb}^{+}$qubit is initially prepared in the state $m_{J}=1 / 2$ (full symbols) or $m_{J}=-1 / 2$ (open symbols). Within a few Langevin collision time periods $t_{L}$ in a gas of $\mathrm{Rb}$ atoms $\left(\left|F=1, m_{\mathrm{F}}=1\right\rangle\right)$ the state relaxes to a mix of $m_{J}= \pm 1 / 2$. $P_{\text {Bright }}$ is the probability of the ion to occupy the state $m_{J}=1 / 2$. Figure adapted from [59].

reached yet. However, first steps in this direction have been taken. For example, the decoherence/ lifetime of an ionic qubit in an atomic bath has been studied [59]. Here, an $\mathrm{Yb}^{+}$ ion is prepared as a single spin impurity in a bath of spinpolarized ${ }^{87} \mathrm{Rb}$ atoms (see Fig.11). Resonant laser light is used to control the population of the two Zeeman sublevels $m_{J}= \pm 1 / 2$ of the ground state of ${ }^{174} \mathrm{Yb}^{+}$. In this way, a qubit is realized and immersed into $\mathrm{Rb}$ atom clouds prepared in the $\left|F=1, m_{\mathrm{F}}=1\right\rangle$ ground state. These first results show a very short coherence lifetime of the qubit. After a few Langevin collisions the ionic state typically relaxes and depolarizes in a spin-nonconserving way. This relaxation mechanism might be due to a very strong spin-orbit coupling which is typical for heavy elements like $\mathrm{Yb}^{+}$and $\mathrm{Rb}$. It will be interesting to see whether such strong spin relaxation can also be seen in other atom-ion systems. From the standpoint of quantum information applications, a system with negligible spin relaxation but efficient spin exchange would be desirable.

\section{Polarons in the strong coupling regime}

When immersing an immobile ionic impurity into a quantum gas, it is expected that atom-ion interaction leads to a local modulation in the quantum gas density [60, 61]. This can be understood in terms of a dressing of the ion with the neutral atoms. If the ion is mobile this dressing gives rise to a quasi-particle, a polaron (see e.g. ref. [62]). Ultracold gases have recently received considerable attention in the context of polaron physics due to the excellent tunability of the relevant parameters, such as the scattering length and the particle density [63, 64, 65, 66, 67, 68, 69, 70].

Of special interest is polaron research in the strong coupling limit where unsolved problems are still present [71]. So far, this limit has been elusive both in condensed matter systems and in systems of cold neutral atoms. However, an ionic impurity with its large interaction range is expected to easily enter the strong coupling regime [71]. Predicted phenomena in this regime include self-trapping and collapse of the condensate. Self-trapping is an especially intriguing phenomenon where the impurity first induces a bubble in the condensate as a consequence of its strong interaction. The bubble retroactively leads to confinement of the impurity.

\section{Mesoscopic molecular ions}

The atom-ion polarons are related to the work in reference [72] where a novel mesoscopic ionic molecule is predicted. This molecule consists of a single ion at rest which loosely binds many neutral atoms into the most weakly bound molecular states of the $1 / \mathrm{r}^{4}$ polarization potential. When placed in a BEC, large shells form around the ion, which are filled with up to hundreds of atoms. The shell size can reach $0.1 \mu \mathrm{m}$ and the binding energy is on the order of 0.1 to $1 \mu \mathrm{K} \times \mathrm{k}_{B}$. For $\mathrm{Na}$, these mesoscopic states are predicted to form spontaneously on the ms timescale (at densities of $10^{14} \mathrm{~cm}^{-3}$ ) after a Na+ ion has been immersed into a Na BEC. The released binding energy is either dissipated by phonon emission in the condensate or by atom-ion three-body collisions. The bound states can in principle decay into more deeply bound states but lifetimes are predicted to be relatively long, on the order of tens of milliseconds. This should make it possible to experimentally investigate some of the properties of these exotic mesoscopic quantum objects.

\section{E. Coupling of crystal phonons to a Fermi gas}

Another recent proposal suggests to combine a cold atomic Fermi gas with a trapped linear ion crystal [73]. The crystal of trapped ions is regarded as an experimentally tunable analog of a solid state lattice. The ultracold gas of fermionic atoms, on the other hand, represents the electron gas of the solid state lattice. The ion crystal and the atom cloud are brought into contact or close to each other $(\approx 100 \mathrm{~nm})$ to allow for interaction. This interaction between atom gas and ion lattice would then correspond to phonon-electron coupling in a solid state phase. If disturbing effects of micromotion can be neglected, such a combined system is potentially able to mimic interesting solid state physics such as polaronic quasiparticles, Peierls transitions, and the emergence of phonon-mediated interactions. The tunability of the ultracold atomic gas combined with the high fidelity operations and detection from trapped ions make this system intriguing when comparing it to possibilities in the solid state phase.

\section{F. Charge transport}

A further idea for cold atom-ion research concerns charge transport mechanisms in an ultracold gas. In ref. [74] a transition in the charge transport mechanism is predicted in a gas of $\mathrm{Na}$ atoms doped with ionic impurities, $\mathrm{Na}^{+}$. At high temperatures charge transport occurs via motion of ions. Collisions of the ions with the gas limit their mobility. At low temperatures 
of a few $\mu \mathrm{K}$, however, charge transport becomes dominated by hopping of electrons from neighboring atoms to the ions. This results in a fast diffusion of the charges through the ultracold gas (of which the motion is then negligible) and the system is expected to exhibit a drastic increase in charge mobility by several orders of magnitude.

\section{SUMMARY}

In conclusion, we have provided an introduction to the young field of cold hybrid atom-ion experiments. After describing the fundamentals of the $1 / \mathrm{r}^{4}$ interaction between an atom and an ion we have given a brief overview of first experiments, ideas for future research as well as challenges to be tackled. At this stage experiments in the field are mostly focussed on elastic and reactive collisions such as charge exchange, quenching processes or molecule formation. These processes are still studied in an energy regime where many partial waves contribute, a fact that can be mainly attributed to residual micromotion of the ion in the Paul trap. Indeed, many ideas for future research hinge on reaching a regime where micromotion can be neglected. Proposals range from applications in quantum information to condensed matter related research. Obviously, as the field of cold atom-ion experiments has just started to unfold, many more routes for research can be envisioned. As an increasing number of scientists are getting involved in this line of research, we are looking forward to an accelerating development and a growing number of exciting results to come.

\section{ACKNOWLEDGEMENTS}

We would like to thank all members of our team for the nice collaboration and discussions over the years. We are also grateful to members of the COMIQ EU Marie Curie training network and the SFB/TRR21 for fruitful exchange. We would like to acknowledge financial support from the German Research foundation DFG within the SFB/TRR21.
[1] D. Leibfried, R. Blatt, C. Monroe, and D.J. Wineland, Quantum dynamics of single trapped ions, Rev. Mod. Phys. 75 (2003), p. 281.

[2] R. Blatt and D. Wineland, Entangled states of trapped atomic ions, Nature 453 (2008), pp. 1008-1015.

[3] H. Häffner, C. Roos, and R. Blatt, Quantum computing with trapped ions, Phys. Rep. 469 (2008), p. 155.

[4] I. Bloch, J. Dalibard, and W. Zwerger, Many-body physics with ultracold gases, Rev. Mod. Phys. 80 (2008), pp. 885-964.

[5] I. Bloch, J. Dalibard, and S. Nascimbene, Quantum simulations with ultracold quantum gases, Nature Physics 8 (2012), p. 267.

[6] A.T. Grier, M. Cetina, F. Oručević, and V. Vuletić, Observation of Cold Collisions between Trapped Ions and Trapped Atoms, Phys. Rev. Lett. 102 (2009), p. 223201.

[7] S. Schmid, A. Härter, and J. Hecker Denschlag, Dynamics of a Cold Trapped Ion in a Bose-Einstein Condensate, Phys. Rev. Lett. 105 (2010), p. 133202.

[8] C. Zipkes, S. Palzer, C. Sias, and M. Köhl, A trapped single ion inside a Bose-Einstein condensate, Nature 464 (2010), p. 388.

[9] R. Côté and A. Dalgarno, Ultracold atom-ion collisions, Phys. Rev. A 62 (2000), p. 012709.

[10] P. Zhang, A. Dalgarno, and R. Côté, Scattering of $Y b$ and $\mathrm{Yb}^{+}$, Phys. Rev. A 80 (2009), p. 030703.

[11] C. Zipkes, L. Ratschbacher, C. Sias, and M. Köhl, Kinetics of a single trapped ion in an ultracold buffer gas, New. J. Phys. 13 (2011), p. 053020.

[12] P. Langevin, Une formule fondamentale de théorie cinétique, (1905).

[13] Z. Idziaszek, T. Calarco, P.S. Julienne, and A. Simoni, Quantum theory of ultracold atom-ion collisions, Phys. Rev. A 79 (2009), p. 010702.

[14] Z. Idziaszek, A. Simoni, T. Calarco, and P.S. Julienne, Multichannel quantum-defect theory for ultracold atom-ion collisions, New Journal of Physics 13 (2011), p. 083005.

[15] K. Ravi, S. Lee, A. Sharma, G. Werth, and S. Rangwala, Combined ion and atom trap for low-temperature ion-atom physics, Applied Physics B 107 (2012), pp. 971-981.
[16] S.J. Schowalter, K. Chen, W.G. Rellergert, S.T. Sullivan, and E.R. Hudson, An integrated ion trap and time-of-flight mass spectrometer for chemical and photo- reaction dynamics studies, Review of Scientific Instruments 83 (2012), p. 043103.

[17] F.H.J. Hall, M. Aymar, N. Bouloufa-Maafa, O. Dulieu, and S. Willitsch, Light-Assisted Ion-Neutral Reactive Processes in the Cold Regime: Radiative Molecule Formation versus Charge Exchange, Phys. Rev. Lett. 107 (2011), p. 243202.

[18] S. Schmid, A. Härter, A. Frisch, S. Hoinka, and J. Hecker Denschlag, An apparatus for immersing trapped ions into an ultracold gas of neutral atoms, Rev. Sci. Instrum. 83 (2012), p. 053108.

[19] C. Zipkes, L. Ratschbacher, S. Palzer, C. Sias, and M. Köhl, Hybrid quantum systems of atoms and ions, J. Phys.: Conf. Ser. 264 (2011), p. 012019.

[20] S. Haze, S. Hata, M. Fujinaga, and T. Mukaiyama, Observation of elastic collisions between lithium atoms and calcium ions, arXiv:1305.3346 (2013).

[21] W.G. Rellergert, S.T. Sullivan, S. Kotochigova, A. Petrov, K. Chen, S.J. Schowalter, and E.R. Hudson, Measurement of a Large Chemical Reaction Rate between Ultracold Closed-Shell ${ }^{40} \mathrm{Ca}$ Atoms and Open-Shell ${ }^{174} \mathrm{Yb}^{+}$Ions Held in a Hybrid AtomIon Trap, Phys. Rev. Lett. 107 (2011), p. 243201.

[22] D.J. Berkeland, J.D. Miller, J.C. Bergquist, W.M. Itano, and D.J. Wineland, Minimization of ion micromotion in a Paul trap, Journal of Applied Physics 83 (1998), pp. 5025-5033.

[23] K. Ravi, S. Lee, A. Sharma, G. Werth, and S. Rangwala, Cooling and stabilization by collisions in a mixed ionatom system, Nat. Comm. 3 (2012), p. 1126.

[24] A. Härter, A. Krükow, A. Brunner, and J. Hecker Denschlag, Minimization of ion micromotion using ultracold atomic probes, Applied Physics Letters 102 (2013), p. 221115.

[25] F. Diedrich, J.C. Bergquist, W.M. Itano, and D.J. Wineland, Laser Cooling to the Zero-Point Energy of Motion, Phys. Rev. Lett. 62 (1989), pp. 403-406.

[26] A. Härter, A. Krükow, A. Brunner, W. Schnitzler, S. Schmid, and J. Hecker Denschlag, Single Ion as a Three-Body Reaction 
Center in an Ultracold Atomic Gas, Phys. Rev. Lett. 109 (2012), p. 123201.

[27] A. Härter, A. Krükow, A. Brunner, and J. Hecker Denschlag, Long-term drifts of stray electric fields in a Paul trap, arXiv:1305.6826 (2013).

[28] C. Raab, J. Eschner, J. Bolle, H. Oberst, F. Schmidt-Kaler, and R. Blatt, Motional Sidebands and Direct Measurement of the Cooling Rate in the Resonance Fluorescence of a Single Trapped Ion, Phys. Rev. Lett. 85 (2000), pp. 538-541.

[29] S. Narayanan, N. Daniilidis, S.A. Möller, R. Clark, F. Ziesel, K. Singer, F. Schmidt-Kaler, and H. Häffner, Electric field compensation and sensing with a single ion in a planar trap, Journal of Applied Physics 110 (2011), p. 114909.

[30] U. Tanaka, K. Masuda, Y. Akimoto, K. Koda, Y. Ibaraki, and $\mathrm{S}$. Urabe, Micromotion compensation in a surface electrode trap by parametric excitation of trapped ions, Applied Physics B 107 (2012), pp. 907-912.

[31] B.L. Chuah, N.C. Lewty, R. Cazan, and M.D. Barrett, Detection of ion micromotion in a linear Paul trap with a high finesse cavity, Opt. Express 21 (2013), pp. 10632-10641.

[32] Q.A. Turchette, D. Kielpinski, B.E. King, D. Leibfried, D.M. Meekhof, C.J. Myatt, M.A. Rowe, C.A. Sackett, C.S. Wood, W.M. Itano, C. Monroe, and D.J. Wineland, Heating of trapped ions from the quantum ground state, Phys. Rev. A 61 (2000), p. 063418.

[33] L. Deslauriers, S. Olmschenk, D. Stick, W.K. Hensinger, J. Sterk, and C. Monroe, Scaling and Suppression of Anomalous Heating in Ion Traps, Phys. Rev. Lett. 97 (2006), p. 103007.

[34] F.G. Major and H.G. Dehmelt, Exchange-collision technique for the rf spectroscopy of stored ions, Phys. Rev. 170 (1968), p. 91.

[35] R. DeVoe, Power-law distributions for a trapped ion interacting with a classical buffer gas, Phys. Rev. Lett. 102 (2009), p. 063001.

[36] M. Cetina, A.T. Grier, and V. Vuletić, Micromotion-Induced Limit to Atom-Ion Sympathetic Cooling in Paul Traps, Phys. Rev. Lett. 109 (2012), p. 253201.

[37] C. Schneider, M. Enderlein, T. Huber, and T. Schätz, Optical trapping of an ion, Nat. Photonics 4 (2010), p. 772.

[38] C. Zipkes, S. Palzer, L. Ratschbacher, C. Sias, and M. Köhl, Cold heteronuclear atom-ion collisions, Phys. Rev. Lett. 105 (2010), p. 12559.

[39] L. Ratschbacher, C. Zipkes, C. Sias, and M. Köhl, Controlling chemical reactions of a single particle, Nat. Phys. 8 (2012), p. 649.

[40] F.H.J. Hall and S. Willitsch, Millikelvin Reactive Collisions between Sympathetically Cooled Molecular Ions and Laser-Cooled Atoms in an Ion-Atom Hybrid Trap, Phys. Rev. Lett. 109 (2012), p. 233202.

[41] S.T. Sullivan, W.G. Rellergert, S. Kotochigova, and E.R. Hudson, Role of Electronic Excitations in Ground-State-Forbidden Inelastic Collisions Between Ultracold Atoms and Ions, Phys. Rev. Lett. 109 (2012), p. 223002.

[42] F.H.J. Hall, P. Eberle, G. Hegi, M. Raoult, M. Aymar, O. Dulieu, and S. Willitsch, Ion-neutral chemistry at ultralow energies: Dynamics of reactive collisions between laser-cooled $\mathrm{Ca}^{+}$ions and Rb atoms in an ion-atom hybrid trap, arXiv:1302.4682 (2013).

[43] F.H.J. Hall, M. Aymar, M. Raoult, O. Dulieu, and S. Willitsch, Light-assisted cold chemical reactions of barium ions with rubidium atoms, Molecular Physics 111 (2013), pp. 1683-1690.

[44] W. Rellergert, S.T. Sullivan, S.J. Schowalter, S. Chen Kuang Kotochigova, and E.R. Hudson, Evidence for sympathetic vibrational cooling of translationally cold molecules, Nature 495 (2013), p. 490.
[45] D. Smith, The ion chemistry of interstellar clouds, Chemical Reviews 92 (1992), pp. 1473-1485.

[46] D. Gerlich and S. Horning, Experimental investigation of radiative association processes as related to interstellar chemistry, Chemical Reviews 92 (1992), pp. 1509-1539.

[47] B. Roth, P. Blythe, H. Wenz, H. Daerr, and S. Schiller, Ionneutral chemical reactions between ultracold localized ions and neutral molecules with single-particle resolution, Phys. Rev. A 73 (2006), p. 042712.

[48] P. Staanum, K. Højbjerre, R. Wester, and M. Drewsen, Probing Isotope Effects in Chemical Reactions Using Single Ions, Phys. Rev. Lett. 100 (2008), p. 243003.

[49] S. Willitsch, M.T. Bell, A.D. Gingell, S.R. Procter, and T.P. Softley, Cold Reactive Collisions between Laser-Cooled Ions and Velocity-Selected Neutral Molecules, Phys. Rev. Lett. 100 (2008), p. 043203.

[50] J. Deiglmayr, A. Göritz, T. Best, M. Weidemüller, and R. Wester, Reactive collisions of trapped anions with ultracold atoms, Phys. Rev. A 86 (2012), p. 043438.

[51] H.F. Hess, D.A. Bell, G.P. Kochanski, R.W. Cline, D. Kleppner, and T.J. Greytak, Observation of Three-Body Recombination in Spin-Polarized Hydrogen, Phys. Rev. Lett. 51 (1983), pp. 483486.

[52] E.A. Burt, R.W. Ghrist, C.J. Myatt, M.J. Holland, E.A. Cornell, and C.E. Wieman, Coherence, Correlations, and Collisions: What One Learns about Bose-Einstein Condensates from Their Decay, Phys. Rev. Lett. 79 (1997), pp. 337-340.

[53] B.D. Esry, C.H. Greene, and J.P. Burke, Recombination of Three Atoms in the Ultracold Limit, Phys. Rev. Lett. 83 (1999), pp. 1751-1754.

[54] A. Härter, A. Krükow, M. Deiß, B. Drews, E. Tiemann, and J. Hecker Denschlag, Population distribution of product states following three-body recombination in an ultracold atomic gas, Nature Physics 9 (2013), pp. 512-517.

[55] Z. Idziaszek, T. Calarco, and P. Zoller, Controlled collisions of a single atom and an ion guided by movable trapping potentials, Phys. Rev. A 76 (2007), p. 033409.

[56] H. Doerk, Z. Idziaszek, and T. Calarco, Atom-ion quantum gate, Phys. Rev. A 81 (2010), p. 012708.

[57] L.H. Nguyên, A. Kalev, M.D. Barrett, and B.G. Englert, Micromotion in trapped atom-ion systems, Phys. Rev. A 85 (2012), p. 052718.

[58] R. Gerritsma, A. Negretti, H. Doerk, Z. Idziaszek, T. Calarco, and F. Schmidt-Kaler, Bosonic Josephson Junction Controlled by a Single Trapped Ion, Phys. Rev. Lett. 109 (2012), p. 080402.

[59] L. Ratschbacher, C. Sias, L. Carcagni, J.M. Silver, C. Zipkes, and M. Köhl, Decoherence of a Single-Ion Qubit Immersed in a Spin-Polarized Atomic Bath, Phys. Rev. Lett. 110 (2013), p. 160402.

[60] P. Massignan, C.J. Pethick, and H. Smith, Static properties of positive ions in atomic Bose-Einstein condensates, Phys. Rev. A 71 (2005), p. 023606.

[61] J. Goold, H. Doerk, Z. Idziaszek, T. Calarco, and T. Busch, Ioninduced density bubble in a strongly correlated one-dimensional gas, Phys. Rev. A 81 (2010), p. 041601.

[62] A. Alexandrov and J. Devreese, Advances in polaron physics, Springer Verlag Springer Series on Solid State Sciences 159 (2009).

[63] F.M. Cuccietti and E. Timmermans, Strong-Coupling Polarons in Dilute Gas Bose-Einstein Condensates, Phys. Rev. Lett. 96 (2006), p. 210401.

[64] R.M. Kalas and D. Blume, Interaction-induced localization of an impurity in a trapped Bose-Einstein condensate, Phys. Rev. A 73 (2006), p. 043608. 
[65] J. Tempere, W. Casteels, M.K. Oberthaler, S. Knoop, E. Timmermans, and J.T. Devreese, Feynman path-integral treatment of the BEC-impurity polaron, Phys. Rev. B 80 (2009), p. 184504.

[66] W. Casteels, T. Cauteren, J. Tempere, and J. Devreese, Strong coupling treatment of the polaronic system consisting of an impurity in a condensate, Laser Physics 21 (2011), pp. 1480-1485.

[67] A. Schirotzek, C.H. Wu, A. Sommer, and M.W. Zwierlein, $O b$ servation of Fermi Polarons in a Tunable Fermi Liquid of Ultracold Atoms, Phys. Rev. Lett. 102 (2009), p. 230402.

[68] S. Nascimbène, N. Navon, K.J. Jiang, L. Tarruell, M. Teichmann, J. McKeever, F. Chevy, and C. Salomon, Collective Oscillations of an Imbalanced Fermi Gas: Axial Compression Modes and Polaron Effective Mass, Phys. Rev. Lett. 103 (2009), p. 170402.

[69] C. Kohstall, M. Zaccanti, M. Jag, A. Trenkwalder, P. Massignan, G. Bruun, F. Schreck, and R. Grimm, Metastability and coherence of repulsive polarons in a strongly interacting Fermi mixture, Nature 485 (2012), p. 615

[70] M. Koschorreck, D. Pertot, E. Vogt, B. Fröhlich, M. Feld, and M. Köhl, Attractive and repulsive Fermi polarons in two dimensions, Nature 485 (2012), p. 619.

[71] W. Casteels, J. Tempere, and J. Devreese, Polaronic Properties of an Ion in a Bose-Einstein Condensate in the Strong-Coupling Limit, Journal of Low Temperature Physics 162 (2011), pp. 266273.

[72] R. Côté, V. Kharchenko, and M.D. Lukin, Mesoscopic Molecular Ions in Bose-Einstein Condensates, Phys. Rev. Lett. 89 (2002), p. 093001.

[73] U. Bissbort, D. Cocks, A. Negretti, Z. Idziaszek, T. Calarco, F. Schmidt-Kaler, W. Hofstetter, and R. Gerritsma, Emulating Solid-State Physics with a Hybrid System of Ultracold Ions and Atoms, Phys. Rev. Lett. 111 (2013), p. 080501.

[74] R. Côté, From Classical Mobility to Hopping Conductivity: Charge Hopping in an Ultracold Gas, Phys. Rev. Lett. 85 (2000), p. 5316. 